\title{
Does a Gradient-Adjusted Cardiac Power Index Improve Prediction of Post-Transcatheter Aortic Valve Replacement Survival Over Cardiac Power Index?
}

\author{
Pradyumna Agasthi ${ }^{1}$, Sai Harika Pujari ${ }^{1}$, Farouk Mookadam ${ }^{1}$, Andrew Tseng ${ }^{2}$, \\ Nithin R. Venepally ${ }^{1}$, Panwen Wang ${ }^{3}$, Mohamed Allam ${ }^{1}$, John Sweeney ${ }^{1}$, Mackram Eleid $^{2}$, \\ Floyd David Fortuin ${ }^{1}$, David R. Holmes Jr${ }^{2}$, Nirat Beohar ${ }^{4}$, and Reza Arsanjani ${ }^{1}$ \\ ${ }^{1}$ Department of Cardiovascular Diseases, Mayo Clinic Arizona, Phoenix, AZ; \\ ${ }^{2}$ Department of Cardiovascular Diseases, Mayo Clinic Rochester, Rochester, MN; \\ ${ }^{3}$ Department of Health Sciences Research, Mayo Clinic Arizona, Scottsdale, AZ; \\ ${ }^{4}$ Columbia University, Division of Cardiology, Mount Sinai Medical Center, Miami Beach, FL, USA.
}

Purpose: Cardiac power (CP) index is a product of mean arterial pressure (MAP) and cardiac output (CO). In aortic stenosis, however, MAP is not reflective of true left ventricular (LV) afterload. We evaluated the utility of a gradient-adjusted CP (GCP) index in predicting survival after transcatheter aortic valve replacement (TAVR), compared to CP alone.

Materials and Methods: We included 975 patients who underwent TAVR with 1 year of follow-up. CP was calculated as (CO× MAP)/[451×body surface area (BSA)] $\left(\mathrm{W} / \mathrm{m}^{2}\right)$. GCP was calculated using augmented MAP by adding aortic valve mean gradient (AVMG) to systolic blood pressure (CP1), adding aortic valve maximal instantaneous gradient to systolic blood pressure (CP2), and adding AVMG to MAP (CP3). A multivariate Cox regression analysis was performed adjusting for baseline covariates. Receiver operator curves (ROC) for CP and GCP were calculated to predict survival after TAVR.

Results: The mortality rate at 1 year was $16 \%$. The mean age and AVMG of the survivors were $81 \pm 9$ years and $43 \pm 4 \mathrm{~mm}$ Hg versus $80 \pm 9$ years and $42 \pm 13 \mathrm{~mm} \mathrm{Hg}$ in the deceased group. The proportions of female patients were similar in both groups $(p=0.7)$. Both CP and GCP were independently associated with survival at 1 year. The area under ROCs for CP, CP1, CP2, and CP3 were 0.67 [95\% confidence interval (CI), 0.62-0.72], 0.65 (95\% CI, 0.60-0.70), 0.66 (95\% CI, 0.61-0.71), and 0.63 (95\% CI 0.58-0.68), respectively.

Conclusion: GCP did not improve the accuracy of predicting survival post TAVR at 1 year, compared to CP alone.

Key Words: Aortic valve stenosis, transcatheter aortic valve replacement, hemodynamics, mortality

\section{INTRODUCTION}

Cardiac power (CP) is defined as the product of simultaneously measured mean arterial pressure (MAP) and cardiac output. ${ }^{1-4}$ By integrating pressure and flow parameters of the cardiovascular system, it is, therefore, an estimate of cardiac function. ${ }^{1}$ In particular, it represents the energy exerted by the left ventricle (LV) to pump blood into the systemic vasculature. Previous studies have shown that cardiac power index (CPI) can be a strong predictor of mortality in chronic heart failure and cardiogenic shock patients. ${ }^{4-9}$

Given the high 1-year mortality rate after transcatheter aor-

Received: February 10, 2020 Revised: April 3, 2020 Accepted: April 11, 2020

Corresponding author: Pradyumna Agasthi, MD, Department of Cardiovascular Diseases, Mayo Clinic Arizona, 5777 E Mayo Blvd, Phoenix, AZ 85054, USA.

Tel: 1-480-301-4072, Fax: 1-480-301-8018, E-mail: pradyumna_agasthi@hotmail.com

The abstract of this study was presented at EuroEcho 2019.

-The authors have no potential conflicts of interest to disclose.

(C) Copyright: Yonsei University College of Medicine 2020

This is an Open Access article distributed under the terms of the Creative Commons Attribution Non-Commercial License (https://creativecommons.org/licenses/by-nc/4.0) which permits unrestricted non-commercial use, distribution, and reproduction in any medium, provided the original work is properly cited. 
tic valve replacement (TAVR), according to the Transcatheter Valve Therapy (TVT) Registry, better risk prediction tools in this population are needed.${ }^{10}$ Previously, we demonstrated an association between resting CP and survival after TAVR, ${ }^{11}$ which is the primary treatment of choice in high risk patients with severe aortic stenosis. ${ }^{12}$ However, MAP does not accurately represent mean systolic left ventricular pressure in patients with aortic stenosis due to the trans-valvular gradient across the aortic valve, which adds to the total $\mathrm{LV}$ afterload. Accordingly, we hypothesized that transvalvular gradient-adjusted CP (GCP) might help to better predict survival post TAVR over CP alone.

In this study, we sought to evaluate the relationship between GCP obtained by three different methods and 1-year mortality in patients with severe aortic stenosis who underwent TAVR.

\section{MATERIALS AND METHODS}

\section{Study population}

This study was conducted as a multicenter retrospective analysis of patients with severe symptomatic aortic stenosis who underwent TAVR between January 1, 2012, and June 20, 2017 at Mayo Clinic hospitals at Phoenix, Arizona; Rochester, Minnesota; and Jacksonville, Florida. The study conformed to institutional guidelines and those of the American Physiological Society. The study included patients aged $\geq 18$ years with symptomatic severe aortic stenosis who underwent TAVR with either balloon expandable or self-expanding aortic valve prostheses, who had a transthoracic echocardiogram (TTE) performed within 2 months of TAVR, and who had follow-up data at 1 year after treatment. Exclusion criteria included patients without follow-up data and patients with moderate to severe aortic regurgitation. Of 1070 patients who underwent TAVR, 95 patients were excluded due to a lack of follow-up data at 1 year, no TTE within 60 days of the index procedure, and refusal to approve research authorization (Supplementary Fig. 1, only online). The Institutional Review Board (IRB) of the Mayo Clinic approved the research protocol (IRB number: 17-010424), and all patients provided research authorization to utilize the medical information. Valve clinic coordinators were responsible for obtaining follow-up data by contacting the patient or the family, and some data were collected from medical provider records at an outside facility. The primary outcome was all-cause mortality at 1 year after TAVR.

\section{Baseline variables}

Pre-procedural variables included age; sex; race; comorbidities, such as diabetes mellitus, hypertension, myocardial infarction, stroke, transient ischemic attack, infective endocarditis, peripheral artery disease, atrial fibrillation/flutter, carotid artery stenosis, or chronic obstructive pulmonary disease; history of smoking, coronary artery bypass graft surgery (CABG), or carotid artery stenting/endarterectomy; heart failure within
2 weeks; New York Heart Association class for the previous 2 weeks; cardiogenic shock within 2 weeks; cardiogenic shock within 24 hours; permanent pacemaker implantation; dialysis; home oxygen use; left main stenosis $\geq 50 \%$; porcelain aorta; vascular access site; type of TAVR valve; serum albumin level; elective versus emergency procedure; type of anesthesia; presence of a mechanical assist device prior to initiation of TAVR; and Society of Thoracic Surgeons-Predicted Risk of Operative Mortality (STS-PROM) score by chart review based on definitions developed by the TVT registry. ${ }^{13}$ We used a manual sphygmomanometer to calculate blood pressure (BP) during TTE according to American College of Cardiology (ACC) guidelines and the following formula: [systolic $\mathrm{BP}+(2 \times$ diastolic $\mathrm{BP})] /(3$ mm of $\mathrm{Hg}$ to calculate MAP) ${ }^{14}$

\section{Echocardiography and its variables}

We used standard ultrasound scanners (Philips iE33; Phillips Medical Systems, Andover, MA, USA; GE Vivid E9, GE Healthcare, Milwaukee, WI, USA) to perform comprehensive Doppler and 2-dimensional TTE on all the patients before undergoing TAVR. The guidelines of European Association of Echocardiography (EAE) and American Society of Echocardiography (ASE) were used to interpret the images. We used ProSolv Cardiovascular Analyzer 3.0 (ProSolv Cardiovascular Inc., Indianapolis, IN, USA) to make offline measurements of the TTE images.

The variables measured for the study included severity of mitral regurgitation, severity of tricuspid regurgitation, left ventricular ejection fraction (LVEF), aortic valve area (AVA), aortic valve systolic mean gradient (AVSMG), right ventricular systolic pressure (RVSP), and cardiac output. Mild, moderate, and severe regurgitation across mitral, tricuspid and aortic valves were assessed based on criteria set forth by the American Society of Echocardiography and Society for Cardiovascular Magnetic Resonance. ${ }^{15}$ Simpson's biplane method of disks was used to measure LVEF. ${ }^{16}$ The continuity equation was utilised to calculate the AVA and indexed to body surface area (AVAI). AVSMG was measured with the modified Bernoulli equation. These parameters were used to classify patients to have severe aortic stenosis and were defined as AVA $\leq 1 \mathrm{~cm}^{2}$, AVAI $\leq 0.6 \mathrm{~cm}^{2}$, and AVSMG $\geq 40 \mathrm{~mm}$ Hg or a peak velocity $\geq 4.0 \mathrm{~m} / \mathrm{s}^{12,17} \mathrm{RVSP}$ was calculated using Bernoulli's equation: $4(\mathrm{~V} 2)+$ right atrial pressure, where $\mathrm{V}$ stands for the tricuspid regurgitant jet peak velocity and right atrial pressure was measured from the diameter of inferior vena cava and its respirophasic changes.$^{18}$ Cardiac output was calculated as [left ventricular outflow tract (LVOT) diameter ${ }^{2} \times 0.785 \times$ LVOT velocity time integral) (liters/minute), in which LVOT diameter was measured in a parasternal long axis view between aortic valve cusp bases and in systole.

$\mathrm{CP}$ was calculated as (MAP $\times$ cardiac output)/[451×body surface area (BSA)] $\left(\mathrm{W} / \mathrm{m}^{2}\right)$ and indexed to body surface area with the use of baseline pre-TAVR echocardiograms to measure $\mathrm{CP}$ $\left(\mathrm{W} / \mathrm{m}^{2}\right)$. GCP was calculated using three different methods: 1 ) $\mathrm{CP1}$, adding aortic valve mean gradient (AVMG) to systolic BP; 
Table 1. Baseline Characteristics

\begin{tabular}{|c|c|c|c|}
\hline Covariates & Alive $(n=840)$ & Deceased ( $n=135)$ & $p$ value \\
\hline Age (yr) & $81 \pm 9$ & $80 \pm 9$ & 0.148 \\
\hline Male sex (\%) & $496(59.04)$ & $83(61.48)$ & 0.637 \\
\hline Caucasian race & $815(97)$ & $130(96.3)$ & $<0.001$ \\
\hline Prior PCl & $305(36.31)$ & $48(35.56)$ & 0.923 \\
\hline Prior CABG & $224(26.67)$ & $42(31.11)$ & 0.298 \\
\hline Prior stroke & $76(9.05)$ & $20(14.81)$ & 0.043 \\
\hline Prior PAD & $421(50.12)$ & 72 (53.33) & 0.516 \\
\hline Smoking history & $24(2.86)$ & $4(2.96)$ & 1.000 \\
\hline Hypertension & 714 (85) & $114(84.44)$ & 0.896 \\
\hline Diabetes mellitus & $294(35)$ & $52(38.52)$ & 0.439 \\
\hline History of infective endocarditis & $7(0.83)$ & $2(1.48)$ & 0.360 \\
\hline Permanent pacemaker & $131(15.6)$ & $25(18.52)$ & 0.378 \\
\hline Previous implantable cardioverter device & $33(3.9)$ & $6(4.44)$ & 0.812 \\
\hline History of transient ischemic attack & $74(8.81)$ & $16(11.85)$ & 0.262 \\
\hline Carotid artery stenosis & $155(18.45)$ & $32(23.7)$ & $<0.001$ \\
\hline Carotid artery stenting/endarterectomy & $55(6.55)$ & $12(8.89)$ & 0.357 \\
\hline Current dialysis & $23(2.74)$ & $12(8.89)$ & $<0.001$ \\
\hline Moderate to severe chronic lung disease & $258(30.72)$ & $63(46.67)$ & $<0.001$ \\
\hline $\mathrm{HmO}_{2}$ & $71(8.45)$ & $24(17.78)$ & 0.001 \\
\hline Atrial fib/flutter & $337(40.12)$ & 79 (58.52) & $<0.001$ \\
\hline Heart failure within 2 weeks & $660(78.57)$ & 120 (88.89) & 0.007 \\
\hline NYHA class within 2 weeks & & & $<0.001$ \\
\hline 1 & $26(3.1)$ & $6(4.44)$ & \\
\hline$\|$ & $178(21.19)$ & $27(20)$ & \\
\hline III & $513(61.07)$ & $75(55.55)$ & \\
\hline IV & $123(14.64)$ & $27(20)$ & \\
\hline Cardiogenic shock within 24 hours & $3(0.36)$ & $0(0)$ & 0.999 \\
\hline Left main stenosis $\geq 50 \%$ & $105(12.5)$ & $22(16.3)$ & 0.217 \\
\hline Proximal left anterior descending artery stenosis $\geq 70 \%$ & $169(20.12)$ & $30(22.22)$ & 0.566 \\
\hline Porcelain aorta & $75(8.93)$ & $15(11.11)$ & 0.423 \\
\hline STS risk score & $8.03 \pm 5.16$ & $9.59 \pm 5.11$ & 0.001 \\
\hline Total albumin & $4.14 \pm 0.38$ & $4 \pm 0.46$ & $<0.001$ \\
\hline Vascular access site & & & 0.076 \\
\hline Femoral & $646(76.9)$ & $96(71.11)$ & \\
\hline Trans-apical & $152(18.1)$ & $30(22.22)$ & \\
\hline Trans-aortic & $29(3.45)$ & $5(3.7)$ & \\
\hline Axillary & $5(0.6)$ & $4(2.96)$ & \\
\hline Subclavian & $5(0.6)$ & $0(0)$ & \\
\hline Trans-iliac & $3(0.36)$ & $0(0)$ & \\
\hline Balloon expandable valve & $656(78.1)$ & $102(75.56)$ & 0.505 \\
\hline Elective procedure & $796(94.76)$ & $122(90.37)$ & 0.049 \\
\hline Anesthesia & & & $<0.001$ \\
\hline Epidural anesthesia & $1(0.12)$ & $0(0)$ & \\
\hline General anesthesia & 722 (85.95) & $122(90.37)$ & \\
\hline Moderate sedation & $117(13.93)$ & $13(9.63)$ & \\
\hline Mechanical assist device in place at start of the procedure & & & $<0.001$ \\
\hline None & $824(98.10)$ & $131(97.04)$ & \\
\hline Catheter-based assist device & $2(0.24)$ & $0(0)$ & \\
\hline Intra-aortic balloon pump & $14(1.67)$ & $4(2.96)$ & \\
\hline Ejection fraction $(\%)$ & $57.22 \pm 12.8$ & $55.74 \pm 14.06$ & 0.221 \\
\hline
\end{tabular}


Pradyumna Agasthi, et al.

Table 1. Baseline Characteristics (continued)

\begin{tabular}{|c|c|c|c|}
\hline Covariates & Alive $(n=840)$ & Deceased (n=135) & $p$ value \\
\hline Aortic valve area $\left(\mathrm{mm}^{2}\right)$ & $0.88 \pm 0.37$ & $0.85 \pm 0.35$ & 0.434 \\
\hline Aortic valve systolic mean gradient $(\mathrm{mm} \mathrm{Hg})$ & $43.24 \pm 13.89$ & $42.1 \pm 12.51$ & 0.371 \\
\hline Moderate to severe mitral valve regurgitation & $37(4.4)$ & $7(5.18)$ & 0.380 \\
\hline Moderate to severe tricuspid valve regurgitation & $58(6.9)$ & $21(15.55)$ & $<0.001$ \\
\hline Moderate pulmonary regurgitation* & $22(2.62)$ & $3(2.22)$ & $<0.001$ \\
\hline Right ventricular systolic pressure (mm Hg) & $41.58 \pm 13.41$ & $45.89 \pm 18.54$ & 0.001 \\
\hline $\mathrm{CP}$ & $0.60 \pm 0.16$ & $0.51 \pm 0.15$ & $<0.001$ \\
\hline CP1 & $0.69 \pm 0.19$ & $0.60 \pm 0.16$ & $<0.001$ \\
\hline CP2 & $0.76 \pm 0.21$ & $0.65 \pm 0.17$ & $<0.001$ \\
\hline СР3 & $0.89 \pm 0.25$ & $0.78 \pm 0.21$ & $<0.001$ \\
\hline
\end{tabular}

PCl, percutaneous coronary intervention; CABG, coronary artery bypass graft surgery; PAD, peripheral artery disease; NYHA, New York Heart Association; STS Society of Thoracic Surgeons; CP, cardiac power.

Data are presented as number (\%) or mean \pm SD.

${ }^{*}$ No patients had severe pulmonary regurgitation.

Table 2. AUC Values for the Parameters

\begin{tabular}{lccccc}
\hline Variable & AUC & AUC $(95 \%$ Cl) & Sensitivity & Specificity & Cut-off \\
\hline CP & 0.6702 & $0.6201-0.7203$ & 0.7103 & 0.6738 & 0.49 \\
CP1 & 0.6542 & $0.6048-0.7037$ & 0.6724 & 0.6549 & 0.59 \\
CP2 & 0.6645 & $0.6165-0.7125$ & 0.6587 & 0.6739 & 0.65 \\
CP3 & 0.6328 & $0.5839-0.6817$ & 0.6314 & 0.6357 & 0.78
\end{tabular}

$\mathrm{AUC}$, area under the curve; $\mathrm{Cl}$, confidence interval; $\mathrm{CP}$, cardiac power.

2) CP2, adding aortic valve maximal instantaneous gradient to systolic BP (CP2); and 3) adding AVMG to MAP (CP3). Due to a lack of published literature on non-invasive assessment of mean left ventricular pressure in patients with aortic stenosis, we made the following assumptions while calculating gradient-adjusted CPI. We assumed that the addition of either mean (CP1) or maximal instantaneous gradient (CP2) across the aortic valve to systemic systolic pressure would likely reflect the true systolic pressure generated by the left ventricular. For the calculation of CP3, we assumed that the addition of mean gradient across the aortic valve to mean systemic pressure would likely reflect the mean left ventricular systolic pressure in one cardiac cycle.

\section{Statistical analysis}

Survival analysis was performed using a step-up technique to identify cut-off values for CPI and gradient-adjusted CPI to recognize where the maximum mortality difference occurred. ${ }^{19}$ The study cohort was divided accordingly into two groups using the cut-off values of $0.49,0.59,0.65$, and $0.78 \mathrm{~W} / \mathrm{m}^{2}$ for $\mathrm{CP}$, $\mathrm{CP1}$, CP2, and CP3, respectively. Continuous variables are shown as means \pm SDs, and categorical variables are presented as numbers and percentages. Baseline characteristics were compared using the t-test for categorical variables and chi-square or Fisher exact tests for continuous variables. Univariate analysis was performed to determine the relationship between baseline variables and all-cause mortality at 1 year with $\mathrm{CP}, \mathrm{CP} 1, \mathrm{CP} 2$, and CP3. All variables with statistically significant associations noted in univariate logistic regression analysis were included

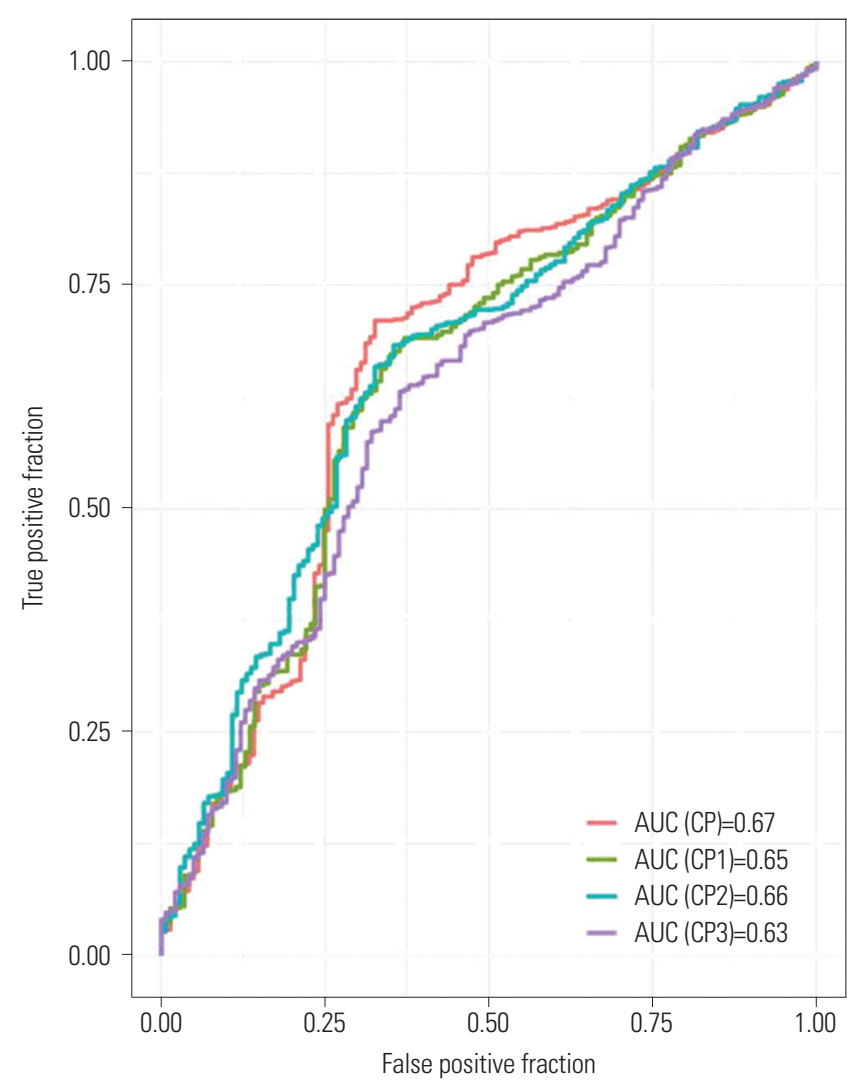

Fig. 1. ROC curves of 1-year mortality prediction with $\mathrm{CP}, \mathrm{CP} 1, \mathrm{CP} 2$, and $\mathrm{CP} 3$ values. $A U C$, area under the curve; $C P$, cardiac power; $\mathrm{ROC}$, receiver operator curves.

in the multivariate survival analysis. Event rates for the study were calculated using the Kaplan-Meier method. Spearman correlation analysis was conducted to assess correlations among $\mathrm{CP}, \mathrm{CP} 1, \mathrm{CP} 2$, and $\mathrm{CP} 3{ }^{20}$

\section{RESULTS}

The study's mean follow-up time was 378 days. The mean age 
of the patients was $81 \pm 9$ years, and $59.04 \%$ were male. All of the baseline characteristics of the study are described in detail in Table 1 and Supplementary Tables 1-4 (only online). Oneyear mortality following the index procedure was $13.94 \%$ (141 patients). CP showed a modest relationship with mortality at 1 year. AUCs of $0.67,0.65,0.66$, and 0.63 were achieved using CP, CP1, CP2, and CP3 respectively (Table 2, Fig. 1). Receiver operating characteristics (ROC) curves analysis for individual parameters are shown in Supplementary Fig. 2 (only online). Fig. 1 demonstrates the ROC curves of all the parameters com-

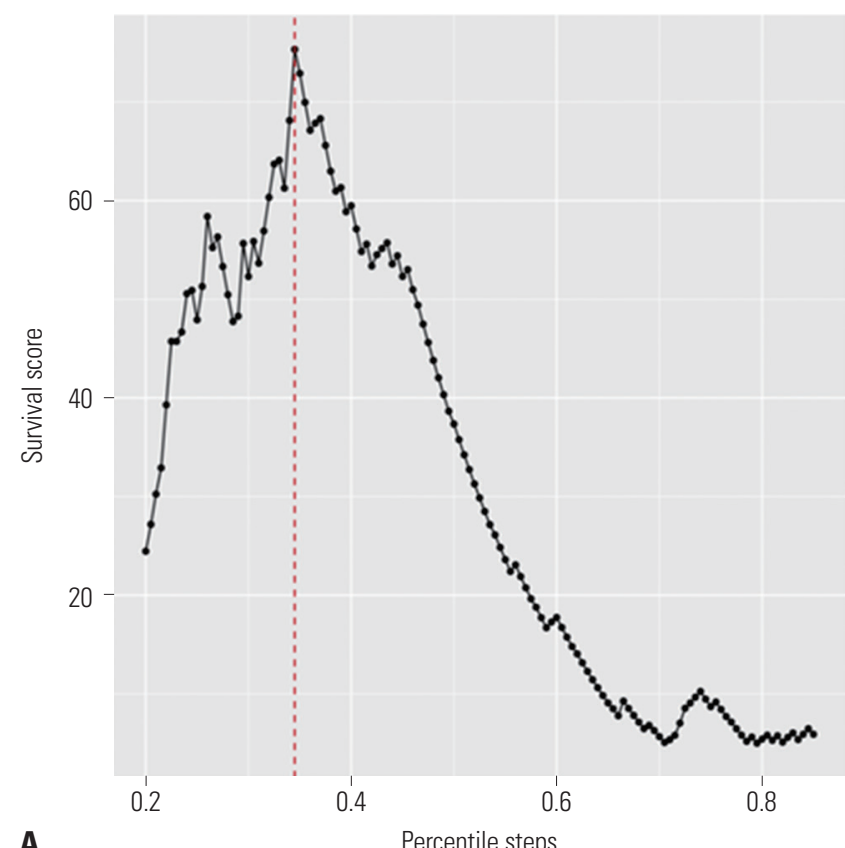

A

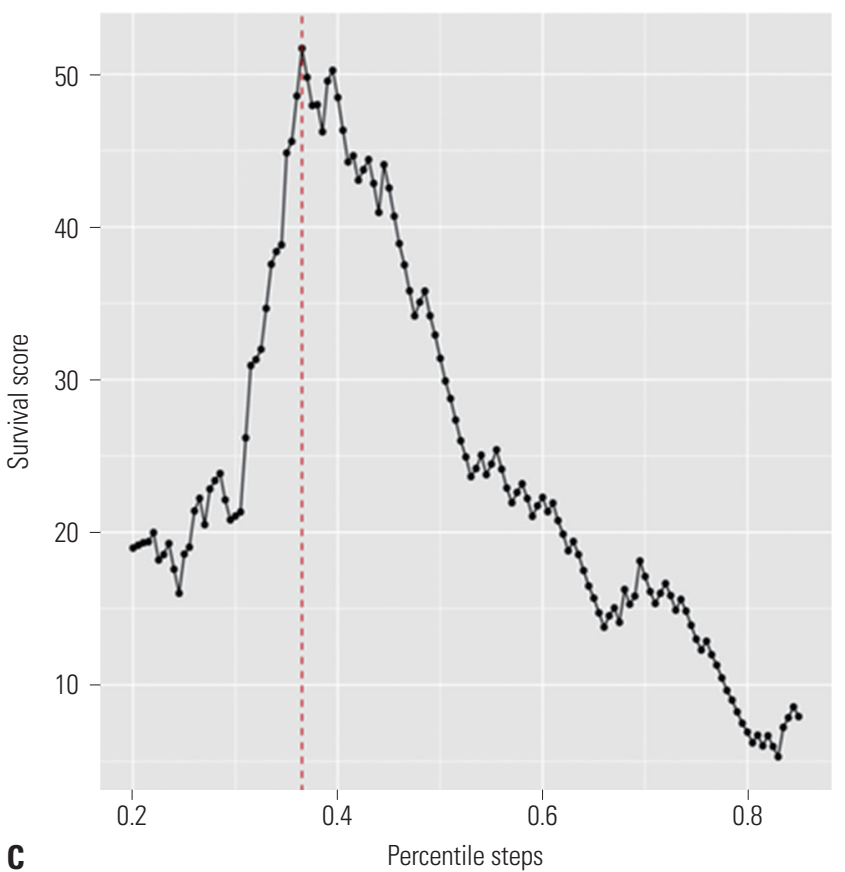

bined. All of the parameters show a significant association with the primary endpoint, with $\mathrm{CP}$ being the strongest (Fig. 1). CPI in the deceased group was significantly lower than in the alive group $\left(0.51 \pm 0.15 \mathrm{~W} / \mathrm{m}^{2}\right.$ vs. $\left.0.6 \pm 0.16, p<0.001\right)$. Gradient-adjusted CPI also showed a significant relationship with patient mortality. CP1, CP2, and CP3 were low in the deceased group: $0.60 \pm$ 0.16 vs. $0.69 \pm 0.19,0.65 \pm 0.17$ vs. $0.76 \pm 0.21$, and $0.78 \pm 0.21$ vs. $0.89 \pm 0.25$, respectively, with $p<0.001$. Patients in the deceased group had a higher prevalence of chronic lung disease, atrial fibrillation/flutter, mild to moderate and severe mitral valve
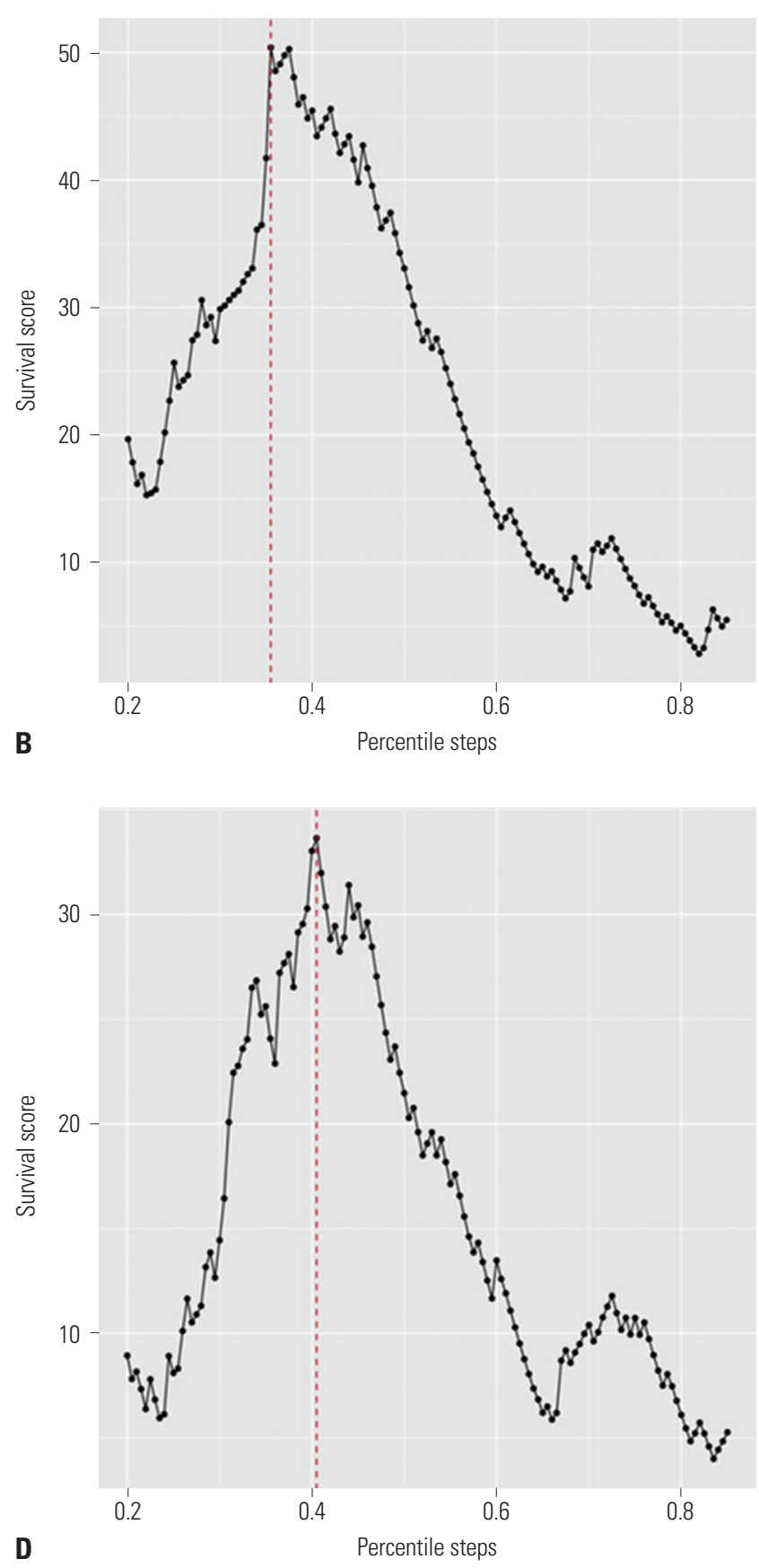

Fig. 2. Survival score analysis to identify cut-offs points for (A) CP, (B) CP1, (C) CP2, and (D) CP3. CP, cardiac power. 
regurgitation, moderate to severe and severe tricuspid valve regurgitation, higher RVSP, and a higher prevalence of patients on dialysis.

Survival score analysis was performed with a step up in the parameters with cut-offs of $0.49,0.59,0.65$, and 0.78 , which produced the maximum distance between the survival curves. Accordingly, the study was divided into groups based on the cut-off values (Fig. 2). Unadjusted Kaplan-Meier survival curves are shown in Fig. $3(p<0.001)$.

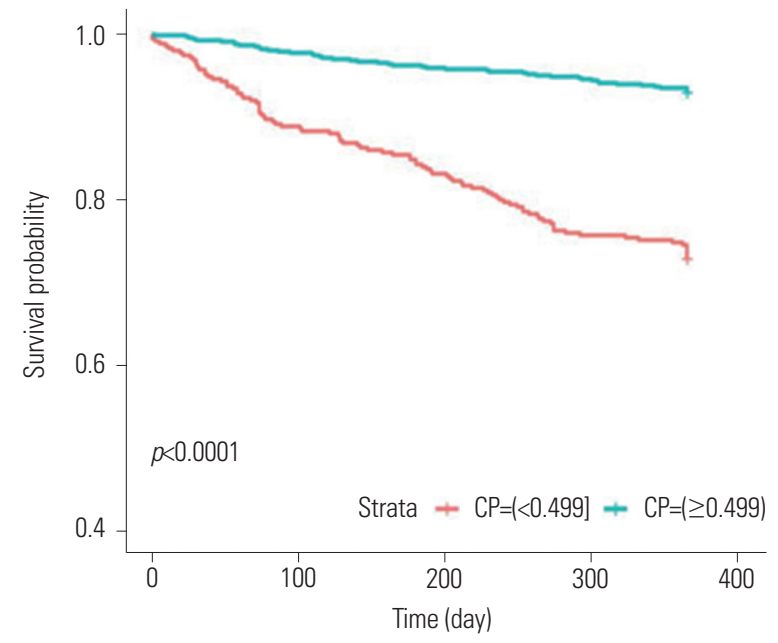

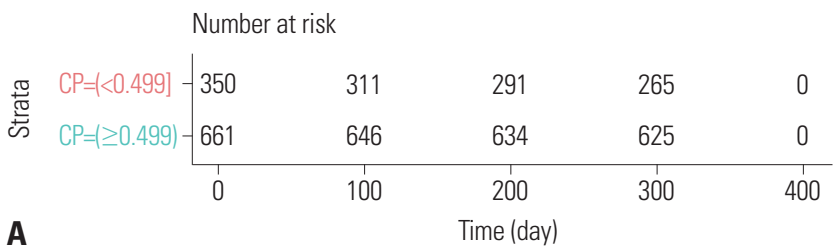

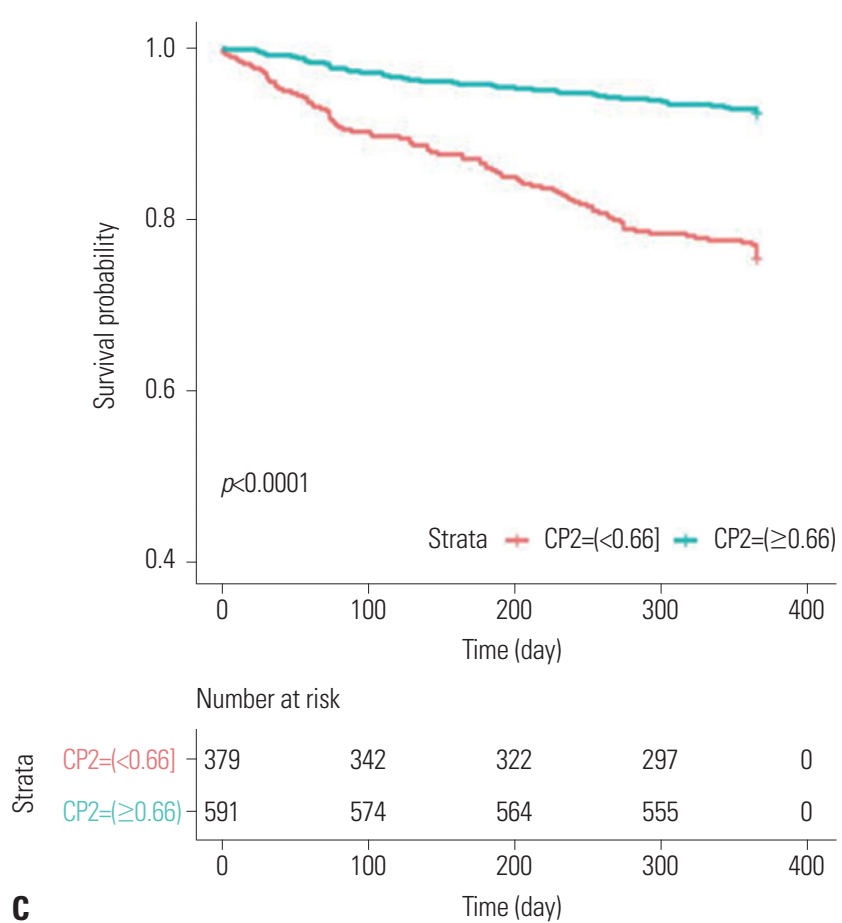

A lower CP was associated with a higher 1-year mortality postprocedure $(24.39 \%$ vs. $8.29 \%, p<0.001) .{ }^{11}$ Subgroup analysis was also performed excluding mitral regurgitation patients, given that the presence of significant mitral regurgitation may underestimate CP. The association remained statistically significant even in the subgroup analysis [hazard ratio, 0.005; 95\% confidence interval (CI), 0.001-0.036, $p<0.001$ ] (Supplementary Figs. 3 and 4, only online). Estimated 1-year mortality as a function of CP, CP1, CP2, and CP3 is shown in Supplementary Fig. 5
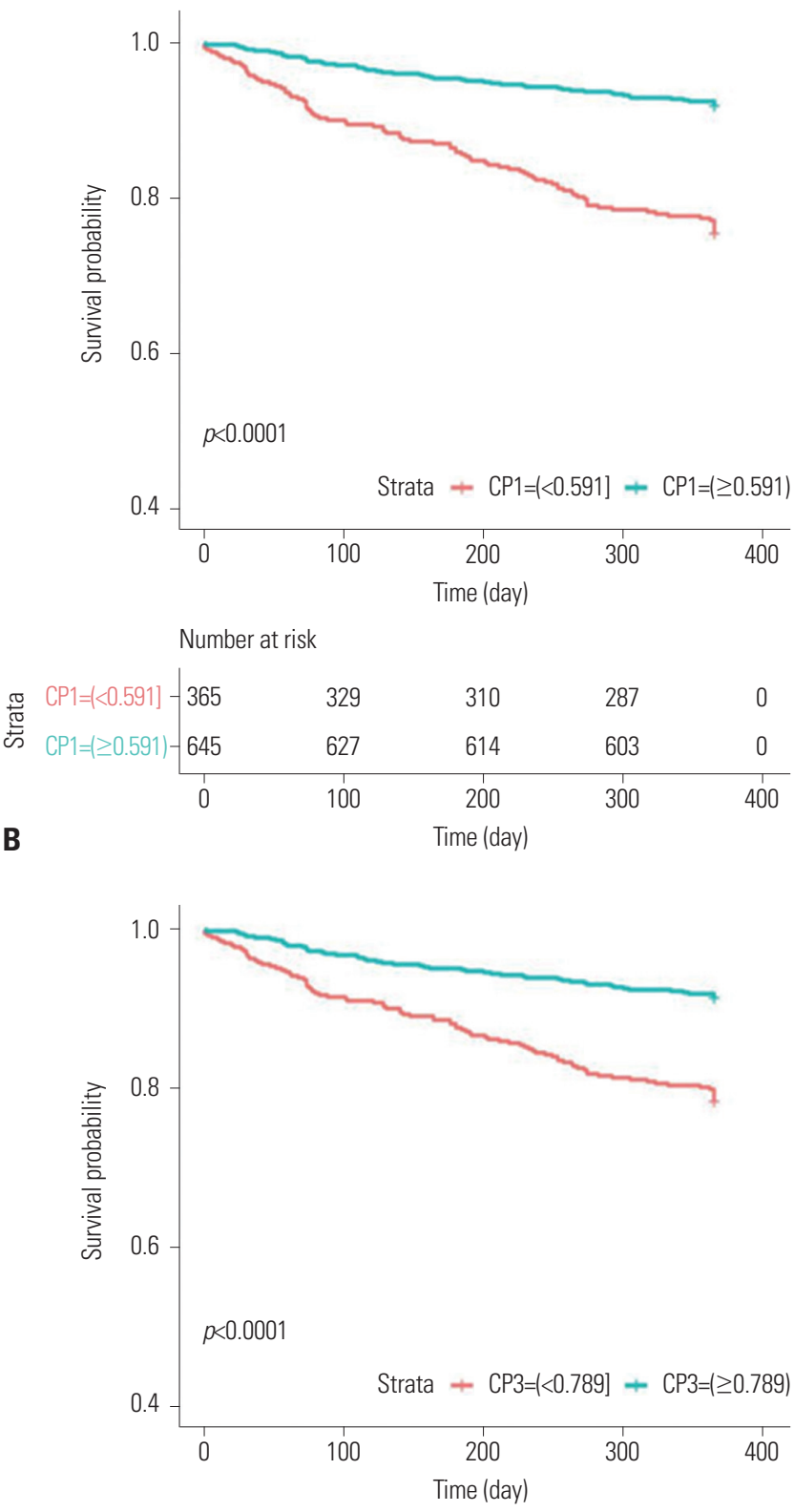

Number at risk

\begin{tabular}{|c|c|c|c|c|c|}
\hline (3) $\quad C P=(<0.789]$ & 414 & 379 & 359 & 337 & 0 \\
\hline$C P 3=(\geq 0.789)$ & 596 & 577 & 565 & 553 & 0 \\
\hline & 0 & 100 & 200 & 300 & 400 \\
\hline
\end{tabular}

Fig. 3. Unadjusted Kaplan-Meier survival curves for (A) CP, (B) CP1, (C) CP2, and (D) CP3. CP, cardiac power. 
Table 3. Univariate Logistic Regression Results of Baseline Variables on 1-Year Mortality

\begin{tabular}{|c|c|c|c|c|}
\hline Covariates & Hazard ratio & 95\% Cl lower & 95\% Cl upper & $p$ value \\
\hline Age (yr) & 0.994 & 0.973 & 1.008 & 0.294 \\
\hline Male sex & 0.938 & 0.668 & 1.315 & 0.709 \\
\hline Prior myocardial infarction & 1.117 & 0.765 & 1.632 & 0.568 \\
\hline Caucasian race & 1.105 & 0.908 & 1.345 & 0.314 \\
\hline Prior percutaneous coronary intervention & 0.968 & 0.684 & 1.368 & 0.852 \\
\hline Prior coronary artery bypass graft & 1.214 & 0.848 & 1.737 & 0.289 \\
\hline Prior stroke & 1.595 & 0.994 & 2.561 & 0.051 \\
\hline Prior peripheral artery disease & 1.132 & 0.814 & 1.576 & 0.461 \\
\hline Smoking history & 1.009 & 0.373 & 2.726 & 0.986 \\
\hline Hypertension & 0.993 & 0.630 & 1.564 & 0.975 \\
\hline Diabetes mellitus & 1.127 & 0.801 & 1.584 & 0.493 \\
\hline History of infective endocarditis & 1.539 & 0.381 & 6.217 & 0.542 \\
\hline Permanent pacemaker & 1.202 & 0.785 & 1.839 & 0.397 \\
\hline Previous implantable cardioverter device & 1.089 & 0.481 & 2.468 & 0.837 \\
\hline History of transient ischemic attack & 1.358 & 0.807 & 2.284 & 0.248 \\
\hline Carotid artery stenosis & 1.118 & 0.939 & 1.331 & 0.209 \\
\hline Carotid artery stenting/endarterectomy & 1.373 & 0.762 & 2.481 & 0.292 \\
\hline Currently on dialysis & 2.694 & 1.491 & 4.868 & $<0.001$ \\
\hline Moderate to severe chronic lung disease & 1.340 & 1.166 & 1.541 & $<0.001$ \\
\hline Home oxygen & 2.073 & 1.336 & 3.216 & $<0.001$ \\
\hline Atrial fibrillation/flutter & 2.000 & 1.431 & 2.797 & $<0.001$ \\
\hline Heart failure within 2-weeks & 2.119 & 1.277 & 3.518 & 0.001 \\
\hline Nyha class within 2-weeks & 1.029 & 0.815 & 1.308 & 0.814 \\
\hline Cardiogenic shock within 24 hours & 0 & 0 & $\operatorname{lnf}$ & 0.583 \\
\hline Cardiac arrest within 24 hours & 0 & 0 & Inf & 0.501 \\
\hline Left main stenosis $\geq 50 \%$ & 1.362 & 0.871 & 2.129 & 0.174 \\
\hline Proximal left anterior descending artery stenosis $\geq 70 \%$ & 1.145 & 0.769 & 1.706 & 0.505 \\
\hline Porcelain aorta & 1.262 & 0.739 & 2.156 & 0.393 \\
\hline Society of thoracic surgeons risk score & 1.032 & 1.014 & 1.051 & $<0.001$ \\
\hline Total albumin (g/dL) & 0.431 & 0.294 & 0.631 & $<0.001$ \\
\hline Vascular access site & 1.143 & 0.925 & 1.413 & 0.215 \\
\hline Balloon expandable valve & 1.112 & 0.753 & 1.642 & 0.593 \\
\hline Elective procedure & 0.494 & 0.289 & 0.843 & 0.008 \\
\hline Anesthesia & 0.734 & 0.443 & 1.226 & 0.237 \\
\hline Mechanical assist device in place at the start of the procedure & 1.264 & 0.764 & 2.091 & 0.357 \\
\hline Ejection fraction (\%) & 0.992 & 0.982 & 1.004 & 0.221 \\
\hline Aortic valve area $\left(\mathrm{mm}^{2}\right)$ & 0.764 & 0.437 & 1.336 & 0.346 \\
\hline Aortic valve systolic mean gradient (mm Hg) & 0.994 & 0.982 & 1.006 & 0.356 \\
\hline Moderate to severe & 1.045 & 0.816 & 1.337 & 0.728 \\
\hline \multicolumn{5}{|l|}{ Mitral regurgitation } \\
\hline Moderate to severe tricuspid regurgitation & 1.449 & 1.221 & 1.721 & $<0.001$ \\
\hline Moderate pulmonary regurgitation* & 1.016 & 0.745 & 1.396 & $<0.001$ \\
\hline Right ventricular systolic pressure (mm Hg) & 1.017 & 1.007 & 1.028 & 0.001 \\
\hline Cardiac power index $\left(\mathrm{W} / \mathrm{m}^{2}\right)$ & 0.027 & 0.008 & 0.093 & $<0.001$ \\
\hline CP1 & 0.030 & 0.016 & 0.057 & $<0.001$ \\
\hline CP2 & 0.066 & 0.037 & 0.117 & $<0.001$ \\
\hline CP3 & 0.252 & 0.158 & 0.401 & $<0.001$ \\
\hline
\end{tabular}

Cl, confidence interval; NYHA, New York Heart Association; CP, cardiac power.

* No patients had severe pulmonary regurgitation. 
(only online).

Univariate logistic regression analysis results of all individual covariates are described in Table 3. Patients with moderate to severe chronic lung disease, requirement of home oxygen, presence of atrial fibrillation, heart failure within 2 weeks of index procedure, higher STS-PROM score, lower serum albumin, emergent TAVR, moderate to severe tricuspid regurgitation, elevated right ventricular systolic pressure, lower CPI, and requirement of dialysis conferred an elevated risk of all-cause mortality at 1 year. Multivariate cox regression analysis was performed with adjustment of baseline variables, including age, sex, race, history of stroke, percutaneous coronary intervention, $\mathrm{CABG}$, peripheral artery disease, hypertension, diabetes mellitus, hemodialysis, $\mathrm{HmO} 2$ use, atrial fibrillation/flutter, STS-PROM score, serum albumin, mitral regurgitation, tricuspid regurgitation, RVSP, and AVSMG to estimate the independent associations of $\mathrm{CP}, \mathrm{CP} 1, \mathrm{CP} 2$, and $\mathrm{CP} 3$ with 1-year mortality post TAVR. All the parameters showed an independent association with elevated 1-year mortality (Table 4).

Spearman correlation curves are illustrated in Fig. 4 to dem-

Table 4. Multivariate Cox Regression Results for 1-Year Mortality

\begin{tabular}{|c|c|c|c|c|}
\hline Covariates & $\begin{array}{l}\text { Hazard } \\
\text { ratio }\end{array}$ & $\begin{array}{l}95 \% \mathrm{CI} \\
\text { lower }\end{array}$ & $\begin{array}{l}95 \% \mathrm{CI} \\
\text { upper }\end{array}$ & $p$ value \\
\hline Cardiac power index $\left(\mathrm{W} / \mathrm{m}^{2}\right)$ & 0.033 & 0.008 & 0.142 & $<0.001$ \\
\hline Cardiac power index* $\left(\mathrm{W} / \mathrm{m}^{2}\right)$ & 0.005 & 0.001 & 0.036 & $<0.001$ \\
\hline CP1 & 0.055 & 0.014 & 0.219 & $<0.001$ \\
\hline $\mathrm{CP} 1^{*}$ & 0.010 & 0.002 & 0.065 & $<0.001$ \\
\hline CP2 & 0.056 & 0.015 & 0.207 & $<0.001$ \\
\hline $\mathrm{CP} 2^{*}$ & 0.009 & 0.001 & 0.055 & $<0.001$ \\
\hline СР3 & 0.115 & 0.037 & 0.356 & $<0.001$ \\
\hline $\mathrm{CP}^{*}$ & 0.032 & 0.007 & 0.142 & $<0.001$ \\
\hline
\end{tabular}

$\mathrm{Cl}$, confidence interval; $\mathrm{CP}$, cardiac power.

Variables included in multivariate analysis: Currently on Dialysis, Moderate to Severe Chronic Lung Disease, Home Oxygen, Atrial Fibrillation/Flutter, Heart Failure within 2-weeks, Society of Thoracic Surgeons Risk Score, Total Serum Albumin, Elective Procedure, Moderate to Severe Tricuspid Regurgitation, Moderate Pulmonary Regurgitation.

*Excluding patients with moderate to severe mitral regurgitation. onstrate the strength of the correlation between $\mathrm{CP}$ and $\mathrm{CP} 1$, $\mathrm{CP} 2$, and CP3. Spearman correlation coefficients between $\mathrm{CP}$, $\mathrm{CP1}$, CP2, CP3, and covariates of age, STS risk score, total albumin, RVSP, and AVSMG are described in Supplementary Tables 5 and 6 (only online). Grouping data of all groups with the best survival cut-offs are shown in Fig. 2. CP1, CP2, and CP3 showed no superiority compared to $\mathrm{CP}$ alone in predicting 1-year mortality post TAVR. ${ }^{20}$

\section{DISCUSSION}

This retrospective cohort study of 975 patients showed that CP and GCP were strongly associated with all-cause mortality at 1 year in patients who underwent TAVR. Survival analysis at 1 year (Supplementary Figs. 3 and 4, only online) showed associations for all parameters with 1-year mortality. CP and GCP associations remained significant with the exclusion of mitral regurgitation patients and multivariate adjustment of baseline demographics, other co-morbidities, echocardiographic, laboratory, and pre-procedural variables. A sub-group analysis excluding mitral regurgitation was performed because cardiac output assessed by echocardiogram in this subgroup may not be accurate with a significant regurgitant volume. All parameters showed a statistical significance even in the subgroup analysis.

Obeying the fundamental Law of Conservation of Energy, the cardiac pump generates and imparts a continuous supply of hydraulic energy to prevent the cessation of circulation, owing to dissipation of energy in the vasculature. ${ }^{21}$ Hence, the cardiac pump converts chemical energy into hydraulic energy in order to maintain a viable circulation. ${ }^{22}$ The complex interaction of vascular compliance and resistance to flow, in addition to intravascular volume and cardiac filling pressures, requires a variable combining flow and pressure to predict outcomes. ${ }^{23}$ CP output, which combines cardiac output and MAP, represents the ability of the heart to deliver hydraulic energy to maintain sufficient circulation, which, when indexed to BSA, gives CPI. Representing the LV expenditure to pump blood into the
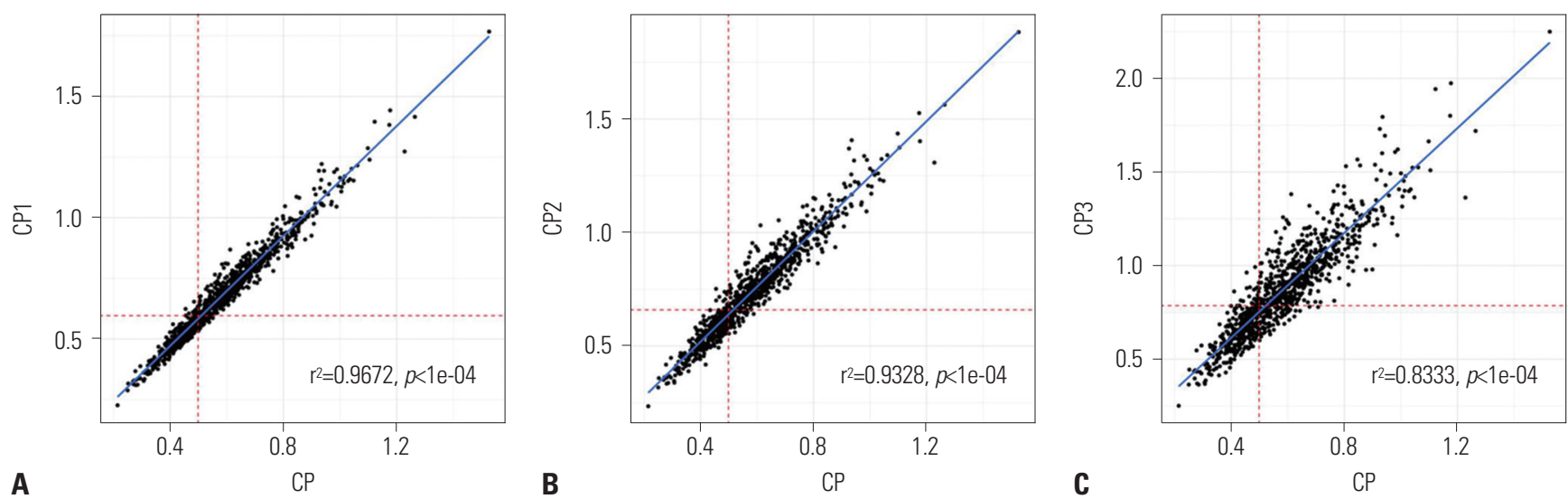

Fig. 4. Spearman correlation graphs comparing $C P$ with $(A) C P 1$, (B) CP2, and (C) CP3. CP, cardiac power. 
systemic vasculature, it provides a more accurate assessment of patient status.

The study's 1-year mortality was $13.84 \%$, which is considerably lower than the US national average of $23.7 \%$ at 1 year. ${ }^{10}$ The mean STS-PROM score was 8.24, compared to the STS/TVT registry score of 7.1 percent. Baseline variables associated with 1-year mortality included chronic lung disease, presence of atrial fibrillation, higher STS-PROM score, the requirement of home oxygen, heart failure within 2-weeks of index procedure, lower serum albumin, moderate to severe tricuspid regurgitation, emergent TAVR, elevated right ventricular systolic pressure, requirement of dialysis, and lower CP. These results corresponded to the results reported by the STS/TVT registry. ${ }^{10}$ Spearman correlation analysis showed a significant association between CP and CP1, CP2, and CP3. ROC curves showed a significant association with 1-year mortality in the study patients (Fig. 4). However, GCP (CP1, CP2, and CP3) was not superior to CPI in estimating mortality post-TAVR.

Previous studies have shown CP to predict outcomes in a variety of other conditions, such as acute coronary syndrome, cardiac arrhythmias, post percutaneous coronary interventions, and cardiogenic shock. ${ }^{4,24-26}$ We previously demonstrated the potential benefit of measuring resting CP to risk stratify patients undergoing TAVR. ${ }^{11}$ We studied 975 patients retrospectively and divided them into two groups by identifying a cut-off value for $\mathrm{CP}$ and concluded that patients with low baseline $\mathrm{CP}\left(\mathrm{CP}<0.48 \mathrm{~W} / \mathrm{m}^{2}\right)$ had higher mortality after 1 year.

MAP, which is used to calculate CP, may not be an accurate reflection of LV afterload in patients with aortic stenosis, because of the presence of the trans-valvular gradient, which increases LV systolic pressure. Hence, we measured GCP by accounting for aortic valve mean gradient and aortic valve maximal instantaneous gradient. Integrating these measures may provide a more accurate assessment of the $\mathrm{CP}$ and could potentially risk stratify the patients undergoing TAVR. However, as the gradient-adjusted CPI did not show superiority when compared to CPI alone in predicting 1-year mortality post TAVR, MAP can be taken to measure the LV pressure to calculate CP.

The pathogenesis and prognoses of aortic stenosis and heart failure are interlinked. A large portion of patients in our cohort had worsening heart failure 2 weeks prior to the procedure. Resting CP for a hemodynamically stable average-sized adult is $1 \mathrm{~W}$, can increase up to $6 \mathrm{~W}$ during exertion and stress, and is significantly diminished in chronic heart failure. ${ }^{6} \mathrm{CP}$ has also been shown to be a useful tool for prognostication in heart failure patients in previous studies. ${ }^{27}$ This is likely attributable to decreasing cardiac functional reserve and aerobic capacity in older aged individuals, with greater reserve less likely to decline compared to low cardiac reserve.

We plan to conduct a subsequent study to evaluate the role of resting $\mathrm{CP}$, peak stress $\mathrm{CP}$, and $\Delta \mathrm{CP}$ to assess relationship with short- and long-term mortality in patients with aortic stenosis undergoing TAVR, focusing on patients with low-flow low-

gradient aortic stenosis.

There can be inherent limitations to a retrospective cohort study, such as study design, selection bias, unmeasured confounders, etc. Several factors, including prevalence of arrhythmias, medical conditions like obesity, and loading conditions, can also affect the accuracy of cardiac output assessed by Doppler echocardiography. A median time gap of 45 days was present before the index procedure and measurement of cardiac output, although, preferably, it should be measured on the same day before the procedure. Hence, it is uncertain whether there was a gradual decline in cardiac function until TAVR was performed. Variability in BP measurement, labile BPs, or temporarily high BPs because of "white coat hypertension" may overestimate/underestimate CP.

In conclusion, gradient-adjusted CPI does not show superiority over CPI alone in predicting 1-year mortality post TAVR. This is likely explained by attenuated CPI noted in patients with severe aortic stenosis. Therefore, incorporation of MAP (measured either invasively/non-invasively) during calculation of CPI without accounting for gradient pressure across the aortic valve would be sufficient for assessing pre-TAVR CPI and predicting mortality post-TAVR.

\section{AUTHOR CONTRIBUTIONS}

Conceptualization: Pradyumna Agasthi, Farouk Mookadam, and Reza Arsanjani. Data curation: Pradyumna Agasthi, Sai Harika Pujari, Andrew Tseng, Nithin R. Venepally, and Mohamed Allam. Formal analysis: Pradyumna Agasthi and Panwen Wang. Investigation: Pradyumna Agasthi, Panwen Wang, Farouk Mookadam, and Reza Arsanjani. Methodology: Pradyumna Agasthi, Panwen Wang, Farouk Mookadam, John Sweeney, Floyd David Fortuin, Mackram Eleid, David R. Holmes Jr, Nirat Beohar, and Reza Arsanjani. Project administration: Reza Arsanjani and Farouk Mookadam. Resources: Mackram Eleid, David R. Holmes Jr, Farouk Mookadam, Reza Arsanjani, and Floyd David Fortuin. Supervision: Reza Arsanjani, Farouk Mookadam, and David R. Holmes Jr. Validation: Pradyumna Agasthi, Panwen Wang, Farouk Mookadam, and Reza Arsanjani. Visualization: Pradyumna Agasthi, Sai Harika Pujari, Andrew Tseng, Nithin R. Venepally, and Mohamed Allam. Writing_original draft: Pradyumna Agasthi and Sai Harika Pujari. Writing - eview \& editing: all authors. Approval of final manuscript: all authors.

\section{ORCID iDs}

Pradyumna Agasthi Sai Harika Pujari

Farouk Mookadam Andrew Tseng Nithin R. Venepally Panwen Wang Mohamed Allam John Sweeney Mackram Eleid Floyd David Fortuin David R. Holmes Jr Nirat Beohar Reza Arsanjani https://orcid.org/0000-0003-3067-6979 https://orcid.org/0000-0002-1073-7482 https://orcid.org/0000-0003-1832-5332 https://orcid.org/0000-0003-4140-3554 https://orcid.org/0000-0002-7156-1927 https://orcid.org/0000-0002-4614-8970 https://orcid.org/0000-0002-7687-9344 https://orcid.org/0000-0002-1981-4106 https://orcid.org/0000-0001-6082-5379 https://orcid.org/0000-0003-2820-6839 https://orcid.org/0000-0002-0037-0373 https://orcid.org/0000-0001-8413-9664 https://orcid.org/0000-0001-7081-4286 


\section{REFERENCES}

1. Cotter G, Moshkovitz Y, Kaluski E, Milo O, Nobikov Y, Schneeweiss A, et al. The role of cardiac power and systemic vascular resistance in the pathophysiology and diagnosis of patients with acute congestive heart failure. Eur J Heart Fail 2003;5:443-51.

2. Cotter G, Moshkovitz Y, Milovanov O, Salah A, Blatt A, Krakover R, et al. Acute heart failure: a novel approach to its pathogenesis and treatment. Eur J Heart Fail 2002;4:227-34.

3. Marmor A, Schneeweiss A. Prognostic value of noninvasively obtained left ventricular contractile reserve in patients with severe heart failure. J Am Coll Cardiol 1997;29:422-8.

4. Williams SG, Cooke GA, Wright DJ, Parsons WJ, Riley RL, Marshall P, et al. Peak exercise cardiac power output; a direct indicator of cardiac function strongly predictive of prognosis in chronic heart failure. Eur Heart J 2001;22:1496-503.

5. Cohen-Solal A, Tabet JY, Logeart D, Bourgoin P, Tokmakova M, Dahan M. A non-invasively determined surrogate of cardiac power ('circulatory power') at peak exercise is a powerful prognostic factor in chronic heart failure. Eur Heart J 2002;23:806-14.

6. Fincke R, Hochman JS, Lowe AM, Menon V, Slater JN, Webb JG, et al. Cardiac power is the strongest hemodynamic correlate of mortality in cardiogenic shock: a report from the SHOCK trial registry. J Am Coll Cardiol 2004;44:340-8.

7. Lang CC, Karlin P, Haythe J, Lim TK, Mancini DM. Peak cardiac power output, measured noninvasively, is a powerful predictor of outcome in chronic heart failure. Circ Heart Fail 2009;2:33-8.

8. Rosenblum H, Helmke S, Williams P, Teruya S, Jones M, Burkhoff D, et al. Peak cardiac power measured noninvasively with a bioreactance technique is a predictor of adverse outcomes in patients with advanced heart failure. Congest Heart Fail 2010;16:254-8.

9. Williams SG, Jackson M, Cooke GA, Barker D, Patwala A, Wright DJ, et al. How do different indicators of cardiac pump function impact upon the long-term prognosis of patients with chronic heart failure? Am Heart J 2005;150:983.

10. Holmes DR Jr, Brennan JM, Rumsfeld JS, Dai D, O’Brien SM, Vemulapalli S, et al. Clinical outcomes at 1 year following transcatheter aortic valve replacement. JAMA 2015;313:1019-28.

11. Agasthi P, Arsanjani R, Mookadam F, Wang P, Venepally NR, Sweeney J, et al. Does resting cardiac power index affect survival post transcatheter aortic valve replacement? J Invasive Cardiol 2020; 32:129-37.

12. Nishimura RA, Otto CM, Bonow RO, Carabello BA, Erwin JP 3rd, Fleisher LA, et al. 2017 AHA/ACC Focused Update of the 2014 AHA/ACC Guideline for the Management of Patients With Valvular Heart Disease: A Report of the American College of Cardiology/ American Heart Association Task Force on Clinical Practice Guidelines. Circulation 2017;135:e1159-95.

13. Carroll JD, Edwards FH, Marinac-Dabic D, Brindis RG, Grover FL, Peterson ED, et al. The STS-ACC transcatheter valve therapy national registry: a new partnership and infrastructure for the introduction and surveillance of medical devices and therapies. J Am Coll Cardiol 2013;62:1026-34.
14. Whelton PK, Carey RM, Aronow WS, Casey DE Jr, Collins KJ, Dennison Himmelfarb C, et al. 2017 ACC/AHA/AAPA/ABC/ACPM/ AGS/APhA/ASH/ASPC/NMA/PCNA Guideline for the Prevention, Detection, Evaluation, and Management of High Blood Pressure in Adults: Executive Summary: A Report of the American College of Cardiology/American Heart Association Task Force on Clinical Practice Guidelines. Hypertension 2018;71:1269-324.

15. Zoghbi WA, Adams D, Bonow RO, Enriquez-Sarano M, Foster E, Grayburn PA, et al. Recommendations for Noninvasive Evaluation of Native Valvular Regurgitation: A Report from the American Society of Echocardiography Developed in Collaboration with the Society for Cardiovascular Magnetic Resonance. J Am Soc Echocardiogr 2017;30:303-71.

16. Lang RM, Badano LP, Mor-Avi V, Afilalo J, Armstrong A, Ernande L, et al. Recommendations for cardiac chamber quantification by echocardiography in adults: an update from the American Society of Echocardiography and the European Association of Cardiovascular Imaging. J Am Soc Echocardiogr 2015;28:1-39.e14.

17. Baumgartner H, Hung J, Bermejo J, Chambers JB, Evangelista A, Griffin BP, et al. Echocardiographic assessment of valve stenosis: EAE/ASE recommendations for clinical practice. J Am Soc Echocardiogr 2009;22:1-23; quiz 101-2.

18. Rudski LG, Lai WW, Afilalo J, Hua L, Handschumacher MD, Chandrasekaran K, et al. Guidelines for the echocardiographic assessment of the right heart in adults: a report from the American Society of Echocardiography endorsed by the European Association of Echocardiography, a registered branch of the European Society of Cardiology, and the Canadian Society of Echocardiography. J Am Soc Echocardiogr 2010;23:685-713; quiz 786-8.

19. Contal C, O'Quigley J. An application of changepoint methods in studying the effect of age on survival in breast cancer. Computational Statistics \& Data Analysis 1999;30:253-70.

20. Spearman C. The proof and measurement of association between two things. Int J Epidemiol 2010;39:1137-50.

21. Tan LB, Williams SG, Tan DK, Cohen-Solal A. So many definitions of heart failure: are they all universally valid? A critical appraisal. Expert Rev Cardiovasc Ther 2010;8:217-28.

22. Hothi SS, Tan LB, Cotter G. Resting cardiac power index and prediction of prognosis in heart failure. Eur J Heart Fail 2015;17:642-4.

23. Grodin JL, Mullens W, Dupont M, Wu Y, Taylor DO, Starling RC, et al. Prognostic role of cardiac power index in ambulatory patients with advanced heart failure. Eur J Heart Fail 2015;17:689-96.

24. Mendoza DD, Cooper HA, Panza JA. Cardiac power output predicts mortality across a broad spectrum of patients with acute cardiac disease. Am Heart J 2007;153:366-70.

25. Tan LB. Cardiac pumping capability and prognosis in heart failure. Lancet 1986;2:1360-3.

26. Tan LB, Littler WA. Measurement of cardiac reserve in cardiogenic shock: implications for prognosis and management. Br Heart J 1990;64:121-8.

27. Cotter G, Williams SG, Vered Z, Tan LB. Role of cardiac power in heart failure. Curr Opin Cardiol 2003;18:215-22. 